\title{
DINOSAURS STILL IN LONG-TERM NET SPECIATION DECLINE BEFORE THE K-PG BOUNDARY
}

\author{
Manabu Sakamoto ${ }^{1}$, Michael J Benton ${ }^{2}$, and Chris Venditti ${ }^{1}$ \\ ${ }^{1}$ School of Biological Sciences, University of Reading, Reading, RG6 6UR \\ ${ }^{2}$ School of Earth Sciences, University of Bristol, Bristol, BS8 1TQ
}

A recent study in Nature Communications ${ }^{1}$ used ecological niche modelling (ENM) to project suitable habitat for dinosaurs during the latest stages of the Cretaceous Period (83-66 million years [Myr] ago) from detailed climate data, apparently refuting the hypothesis that dinosaurs were in a long-term decline ${ }^{2}$ before the Cretaceous-Paleogene ( $\mathrm{K}-\mathrm{Pg}$ ) mass extinction event. However, we note here that: 1 ) suitable habitat does not necessarily equate to taxonomic diversity (i.e., number of species); and 2) lack of a decline in diversity across time intervals is not evidence against a gradual decline in net speciation through time $^{2}$ - diversity and net speciation measure two separate things. Diversity measures the number of species within discrete time intervals, while phylogenetically inferred net speciation measures the dynamic relationship between speciation and extinction through time. Additionally, net speciation is estimated in a model framework that accounts for statistically undesired effects of shared ancestry, whereas diversity is not. Thus, diversity and net speciation are not directly comparable, and a lack of a decline in diversity does not necessarily refute a decline in net speciation through time.

While the ENM implemented by Chiarenza et al. ${ }^{1}$ is impressive and we have no reason to question the accuracy of their model, linking the projected suitable habitat with species diversity presents a slight problem. That is, even though a particular region of geography may represent a suitable habitat for a particular clade, that does not prove that it was inhabited by the target species for any number of reasons - e.g., geographical barriers, low dispersal rates, etc. On the other hand, even if those suitable habitats were inhabited by constituent species of a target clade, they may in fact be occupied by the same species as those already known from the fossiliferous regions, thus not increasing the number of unique species for that time interval. For instance, the projected habitats for the Maastrichtian may be inhabited by Tyrannosaurus rex instead of a different tyrannosaur species that is currently unknown. This is highly likely especially if net speciation were low, and this gives a situation with a low diversity of species but high abundance of individuals that had wide geographical ranges, which is frequently observed in North American dinosaur taxa/clades of the latest Cretaceous. Regardless of which scenario is most likely, the presence of additional regions of suitable habitat does not necessarily equate to an increase in diversity.

More crucially, diversity (= species richness) as implied by Chiarenza et al. ${ }^{1}$ from their ENM, equates to the numbers of taxa within distinct intervals of time - e.g., geological stages. This is regardless of whether various sources of bias have been accounted for - e.g., through the use of rarefaction, $\mathrm{SQS}^{3}$, TRiPS $^{4}$, or spatial sampling standardization ${ }^{5}$. Plots of diversity through time (DTT plots) are then straightforward comparisons of the numbers of taxa across time intervals. Phylogenetic estimates of net speciation on the other hand are used to measure the net increase/decrease in speciation - commonly represented as the difference between speciation $(\lambda)$ and extinction $(\mu)$ rates - and how that relationship may change through time. Net speciation is, in one way or another, related to a measure of the 
numbers of nodes or branches with respect to time and is not based directly on estimates of species diversity (tips on a phylogenetic tree) at any point in time.

Furthermore, net speciation as inferred by Sakamoto et al. ${ }^{2}$ is not equivalent to changes in the number of species across time intervals - i.e., the peaks and troughs of a DTT plot. Sakamoto et al. ${ }^{2}$ regressed the number of nodes separating each tip from the root of the phylogeny (node count) against the time elapsed since the root (time), in a phylogenetic framework accounting for phylogenetically-structured variance-covariance associated with shared ancestry. That is, closely related species are expected to have more similar node count values compared to more distantly related species - indeed sister taxa have identical node counts. Another way to view this is by considering the probability of observing a speciation event at different parts of the tree. Sections of the phylogeny where there are more lineages will necessarily be associated with a higher probability of observing a speciation event. That is, there is an underlying expectation that speciose sections of the phylogenetic tree are associated with high node counts compared to other parts of the tree, in proportion to phylogenetic structure. Our phylogenetic generalized linear mix model (GLMM) accounts for this effect of shared ancestry, by effectively weighting node counts by the phylogenetic variance-covariance. Thus, the inferred pattern of a long-term decline in net speciation through time ${ }^{2}$ is the product of phylogenetic 'correction' and is not directly comparable to changes in species diversity.

In conclusion, the projected areas of suitable habitats from ENMs do not necessarily equate to an increase in species diversity. Even if one were to argue that species diversity remained high throughout the Late Cretaceous to the K-Pg boundary - i.e. no decline in the number of species - this does not mean that the underlying process of speciation and extinction was not associated with a long-term decline in net speciation. The latter, determined from node count accounting for the statistical effects of shared ancestry, is not directly comparable to changes in the number of species through time. Thus, demonstrating that the numbers of species did not decline across time intervals does not refute the phylogenetically-inferred decline in net speciation through time.

\section{REFERENCES}

1. Chiarenza AA, et al. Ecological niche modelling does not support climatically-driven dinosaur diversity decline before the Cretaceous/Paleogene mass extinction. Nat Commun 10, 1091 (2019).

2. Sakamoto $\mathrm{M}$, Benton MJ, Venditti C. Dinosaurs in decline tens of millions of years before their final extinction. Proceedings of the National Academy of Sciences 113, 5036-5040 (2016).

3. Alroy J. Fair Sampling of Taxonomic Richness and Unbiased Estimation of Origination and Extinction Rates. The Paleontological Society Papers 16, 55-80 (2010).

4. Starrfelt J, Liow LH. How many dinosaur species were there? Fossil bias and true richness estimated using a Poisson sampling model. Philos Trans $R$ Soc Lond B Biol Sci 371, 20150219 (2016). 
5. Close RA, Benson RBJ, Upchurch P, Butler RJ. Controlling for the species-area effect supports constrained long-term Mesozoic terrestrial vertebrate diversification. Nat Commun 8, 15381 (2017).

\section{COMPETING INTERESTS}

We declare no competing interests.

\section{AUTHOR CONTRIBUTIONS}

MS, MJB and CV contributed equally to this manuscript. 